\title{
ET search project in struggle for survival
}

Boston. After the demise of the National Aeronautics and Space Administration's (NASA's) Search for Extraterrestrial Intelligence (SETI) programme last year, and growing threats to efforts at Ohio State University (see Nature 374, 668; 1995), a project that has been run by the University of California, Berkeley, at Arecibo, Puerto Rico for the past three years may be the next victim of the US Congress' 1994 decision to cancel SETI funding.

"We're living on our residual NASA funds," says Stuart Bowyer, principal investigator for SERENDIP, a search that has been carried out at various locations since 1979. "That will keep us going for about six months, but then we'll have to stop unless we find some other means of support."

SERENDIP has been supported by NASA virtually from the beginning. "We know how to write grant proposals, but we don't know the first thing about fund-raising," says Dan Werthimer, programme manager for the project. "We're still struggling with that." A non-profit group, known as the Friends of SERENDIP, was formed in mid-1994 to handle contributions from private donors. But donations have been slow to arrive.

Ironically, the financial difficulties coincide with a major upgrade that will transform the present SERENDIP III system to the next generation, SERENDIP IV, creating a 40 -fold increase in search capability. The new instrumentation is due to be installed by September, and will be able to inspect 174 million radio channels every 1.7 seconds, compared to the current 4.2 million channel system.

"We have enough money to get SERENDIP IV installed," says Werthimer. "It will be a shame to get that done and then have to shut down for lack of funds."

The frugality of the programme may be its salvation, as the Berkeley team needs only about $\$ 65,000$ a year to stay afloat. In contrast, the Phoenix project - part of the defunct NASA programme that was rescued by the non-profit SETI Institute and moved from Arecibo to the Parkes radio antenna in New South Wales, Australia - spends nearly 100 times as much.

Although SERENDIP is one of the most powerful SETI searches on the planet, it can get by on the cheap thanks to a neat design concept called 'piggybacking'. SERENDIP basically gets a free ride at Arecibo, operating alongside conventional radioastronomy observations without interfering with them.

While Phoenix scientists have to buy telescope time in order to pick and choose the stars in the sky they want to examine, SERENDIP is more like an eavesdropping device that listens in on radio 'conversations' other astronomers have already tuned into.
"It's an extremely cost-effective approach, because observing time at Arecibo is hard to come by and is also very expensive," says Mike Davis, project scientist at Arecibo. "They're one of the few programmes to get continuous telescope time here."

This is no small matter given that Arecibo is the largest radiotelescope in the world. By linking up to its 1000 -foot diameter dish,

\section{IMAGE UNAVAILABLE FOR COPYRIGHT REASONS}

Sullivan, a radioastronomer at the University of Washington, Seattle. "It's like a blindfolded guy mowing the lawn. Eventually he'll get most of it cut, save for a few tufts, even if he goes over it in a haphazard way."

Sky coverage is not the only objective, as searching for peculiar radio signals is really just half the game in SETI today. The other equally critical task is the steady improve-

$\rightarrow$ ment in technology that may ultimately

o lead to success. The SERENDIP team

has been a leader on this front, provid-

in ing the receiver used in the Ohio State

search, as well as the general architec-

ture for the spectrometer to be

installed in BETA, the new SETI sys-

产 tem designed by the Harvard physicist

? Paul Horowitz.

SERENDIP's biggest contribution, however, may be in introducing the piggybacking approach to radioastronomy, paving the way for similar efforts, either planned or in progress. A pulsar search led by Berkeley and the University of Cornell, which relies on this technique, is now under way at Arecibo, along with a spectral search for hydrogenthat has been designed by Horowitz and scientists from Massachusetts Institute of Technology.

Horowitz has also developed a spectrometer with colleagues at Cornell that will soon be deployed in another piggyback-style pulsar search at Arecibo. "There are many other projects that could run in this fashion - basically searches that do not target specific places in the sky," Davis comments.

Steve Nadis

\section{Big four dominate Australian research}

Sydney. Just four of Australia's 34 universities account for nearly half of the research effort in the nation's higher education sector, according to statistics released last week.

The figures have been collected by the Australian Vice-Chancellors Committee, and represent the first detailed figures on university research funding in Australia as well as a measure of the domination of the sector by a handful of institutions.

Of the \$A524 million (US\$390 million) spent on research by universities during 1993, the University of Melbourne accounted for $\mathbf{1 2 . 1}$ per cent, followed by the Universities of New South Wales (11.5 per cent), Sydney (11.1 per cent), and Queensland (10.5 per cent). These four older bodies were promptly dubbed the "sandstone club" by the media, a reference to the popular building material of colonial times.

Most of the remaining research funds were allocated to a clear second tier of institutions. These include the Universities of Monash (7.9 per cent), Adelaide (6.2), Western Australia (6.1), and Flinders University in South Australia (3.1).

Judged in terms of publications alone (including book chapters, review articles and patents) Monash topped the rankings with 11.9 per cent of research output. In contrast, Sydney came first in the ranking of completed higher research degrees shows, with 344 completed masters and doctorate courses in 1992, and Melbourne came second with 304 .

A comparison of the 1993 figures with those of the previous year show that research funding increased 11 per cent in 1993 with support from the private sector increasing 16 per cent to $\$ 126$ million. Frank Hambley, executive director of the vice-chancellors committee, said that the latter had been partly the result of a new co-operative research centre programme which had emphasized participating by industry.

Mark Lawson 